\title{
Liderazgo directivo en escuelas y proyectos no formales en centros de menores y carcelarios de la Región Metropolita de Chile
}

\section{Managerial leadership in schools and non-formal projects in juvenile and prison centers in the Metropolitan Region of Chile}

DOI: $10.46932 / \mathrm{sfjdv2n5-061}$

Received in: Oct 1st, 2021

Accepted in: Dec 30th, 2021

\author{
Marcela Gaete Vergara \\ Dra. Educación \\ Facultad de Filosofía y Humanidades \\ Universidad de Chile \\ Ana Frank. 582. Quilicura. Santiago. Chile \\ E-mail: magaete@uchile.cl \\ Violeta Acuña Collado \\ Dra. Educación \\ Facultad de Educación \\ Universidad de Playa Ancha \\ Avenida Playa ancha 850. Valparaíso. Chile \\ E-mail: v-acuna@upla.cl \\ Marisol Ramírez Muga \\ Mg. Género y Cultura en América Latina \\ Facultad de Filosofía y Humanidades \\ Universidad de Chile \\ Capitán Ignacio Carrera Pinto. 1025, Ñuñoa. Santiago. Chile \\ E-mail: solmuga@uchile.cl
}

\section{RESUMEN}

La educación en contextos encierro es un tema poco estudiado e incluso invisibilizado, los/as docentes y los equipos directivos no cuentan con apoyos específicos suficientes para llevar a cabo su labor, la que está sujeta a una serie de condiciones y demandas muy diferentes a la del medio libre. Una investigación cualitativa en curso, en que se han recogido datos del $70 \%$ de los participantes, cuyo propósito es cartografiar el sentido los proyectos formativos con jóvenes y adultos en contextos de encierro en la R.M de Chile, arroja información relevante en torno al liderazgo directivo. En todos los casos los principios que guían los proyectos educativos constituyen parte del "sueño pedagógico" de los equipos que conducen las escuelas o programas, quienes se caracterizan por un fuerte compromiso ético y por una toma de decisiones que privilegia el desarrollo de las capacidades de los/as internos/as. Consecuentemente, los equipos directivos no dudan en ir más allá de lo que les corresponde y buscar alternativas autogestionadas para realizar su labor. Lo anterior posibilita que los proyectos no formales y las escuelas se constituyan en un espacio simbólico muy diferente al espacio carcelario, alejándose de la lógica de vigilar y castigar.

Palabras claves: Liderazgo directivo, contextos encierro, escuelas en cárceles, educación penitenciaria 


\begin{abstract}
Education in confinement contexts is a little studied and even invisibilized topic; teachers and management teams do not have enough specific support to carry out their work, which is subject to a series of conditions and demands very different from those of the free environment. A qualitative research in progress, in which data have been collected from $70 \%$ of the participants, whose purpose is to map the meaning of educational projects with young people and adults in confinement contexts in the R.M. of Chile, yields relevant information on managerial leadership. In all cases, the principles guiding the educational projects are part of the "pedagogical dream" of the teams leading the schools or programs, who are characterized by a strong ethical commitment and by decision-making that favors the development of the inmates' capacities. Consequently, the management teams do not hesitate to go beyond their responsibilities and seek self-managed alternatives to carry out their work. This makes it possible for non-formal projects and schools to become a symbolic space that is very different from the prison space, moving away from the logic of surveillance and punishment.
\end{abstract}

Keywords: Managerial leadership, prison contexts, schools in prisons, prison education.

\title{
1 INTRODUCCIÓN
}

En el presente escrito presentamos parte de los resultados de una investigación FONDECYT titulada "Cartografía Analítica de los proyectos formativos con jóvenes y adultos en contextos de encierro de la Región Metropolita (R.M) de Chile”. Dicha investigación cualitativa en una primera etapa, actualmente finalizada, identifica los proyectos formativos formales (escuelas) y no formales que se implementan entre los años 2017 y 2018 en Centros del Servicio Nacional de Menores (SENAME) y Cárceles de la R.M.

La identificación de los proyectos no formales ha sido del todo compleja pues no hay un registro de estos en ninguna instancia oficial centralizada, encontrándose muchos de ellos invisibilizados. Ello, porque sus implementaciones siguen diversas vías: algunos proyectos son implementados a través de licitación pública llamada por SENAME o Gendarmería de Chile, otros, corresponden a agrupaciones que trabajan con fondos propios y realizan convenios directos con SENAME o Gendarmeria Regional, también se encuentran algunas agrupaciones de voluntarios o de estudiantes, funcionarios y/o docentes universitarios que realizan un trabajo pedagógico desde el rol social y político con las que las universidades están comprometidas, en estos casos, las relaciones son directamente con cada uno de los Centros. A su vez, las escuelas también realizan alianzas con universidades para la realización de prácticas u otros proyectos que dan lugar a otra gama de proyectos formativos en contextos de encierro. Así, en un mismo Centro transitan personas e instituciones que sin conocerse entre sí, y sin ni siquiera saber de la existencia de los otros, trabajan, muchas veces, con los mismos internos e internas.

La segunda etapa de la recogida de datos consiste en entrevistar a los equipos directivos de los proyectos y analizar documentos, si los hubiere, que plasmen sus orientaciones formativas. La tercera 
etapa, incluye talleres de análisis cartográfico con los equipos de docentes y directivos que se proyecta para fines del año 2018.

Este escrito presenta un análisis a partir de 32 entrevistas realizadas a equipos directivos de los proyectos, ya sea director, subdirector, Jefe UTP (en caso de escuelas), coordinador u otro cargo directivo equivalente. Si bien, desde el diseño de la investigación no se consideró como eje de análisis el liderazgo de los equipos directivos sino la orientación pedagógica de los proyectos, creemos que es relevante visibilizar esta dimensión, pues nos encontramos con una realidad particular, con sus propias problemáticas, desafíos y necesidades que no ha sido materia de investigación, a la luz de escasa bibliografía sobre liderazgo directivo en instituciones escolares en contextos carcelarios.

Tabla $\mathrm{N}^{\circ} 1$

$\mathrm{N}^{\circ}$ de directores entrevistados según dependencia

\begin{tabular}{c|l}
\hline $\begin{array}{c}\mathbf{N}^{\circ} \text { de directivos } \\
\text { Entrevistados. Julio 2018 }\end{array}$ & \multicolumn{1}{c}{ DEPENDENCIA INSTITUCIONAL } \\
\hline $\mathbf{4}$ & $\begin{array}{l}\text { Escuela SENAME. } \\
\text { 2 municipales y 2 particulares subvencionadas. }\end{array}$ \\
\hline $\mathbf{6}$ & Escuelas en Cárceles Municipales \\
\hline $\mathbf{2}$ & ONG \\
\hline $\mathbf{8}$ & Fundaciones y Corporaciones \\
\hline $\mathbf{1}$ & Colectivos \\
\hline $\mathbf{7}$ & $\begin{array}{l}\text { Carreras Educación Superior Técnica o } \\
\text { universitaria. Línea de prácticas }\end{array}$ \\
\hline $\mathbf{5}$ & $\begin{array}{l}\text { Proyectos Universitarios de Extensión y/o } \\
\text { voluntariados }\end{array}$ \\
\hline
\end{tabular}

Un resultado relevante, que pondremos en discusión, es que más allá de las diferencias entre los tipos de proyectos formales y no formales en relación con sus objetivos, compromisos ideológicos, políticos o religiosos, financiamiento, visibilización, posicionamiento, estructura, gestión, autonomía de trabajo, etc., observamos dimensiones comunes en la forma en que los directivos refieren a su trabajo, sus motivaciones, sus prácticas, su compromiso, entre otros.

En todos los casos la fuente y motor de su práctica directiva está constituida por lo que llamaremos un "sueño pedagógico", que atraviesa su discurso y las prácticas que lo concretizan. Dicho sueño está caracterizado por un fuerte compromiso moral (en ocasiones cristiano) y ético y la férrea creencia en la educación como derecho humano y único motor para la justicia social. Consecuentemente, los equipos directivos no dudan en ir más allá de lo que les corresponde y buscar alternativas autogestionadas para realizar su labor. Lo anterior posibilita que los proyectos no formales y las escuelas se constituyan en un espacio simbólico muy diferente al espacio carcelario, alejándose de la lógica de vigilar y castigar.

El análisis se estructura en dos partes. La primera, destinada a un análisis crítico del Marco para la Buena Dirección y Liderazgo Escolar, en adelante MBDLE, como único instrumento que rige las prácticas directivas en escuelas en contextos de encierro. La segunda, intenta relevar las prácticas 
directivas comunes entre directivos de escuelas y proyectos no formales, las que no obedecen a una perspectiva gerencial del liderazgo, como se infiere del MBDLE, sino a una perspectiva social de la práctica directiva.

\title{
2 MARCO PARA EL LIDERAZGO ESCOLAR EN CHILE. SU (IM)PERTINENCIA EN CONTEXTOS DE ENCIERRO
}

Desde la década de los 90, las políticas educativas en Chile han mostrado una creciente preocupación por establecer marcos comunes para regular el desempeño de los equipos directivos de las escuelas. En esta línea se pueden encontrar dos tipos de medidas, una tendiente a estandarizar la práctica directiva y otra, relacionada con la idoneidad de quienes ejercen las funciones.

\begin{abstract}
Por una parte, [se encuentran] un conjunto de leyes e iniciativas que buscan definir las responsabilidades y atribuciones asociadas a los cargos directivos, con el objetivo de orientar su práctica dentro de la escuela (y hacia fuera de ella) y generar las condiciones para su ejercicio. Acá se encuentran las iniciativas que definen las responsabilidades o ámbitos de acción asociados a la función y que fijan las atribuciones para su ejercicio. Por otro, se han desarrollado un conjunto de iniciativas que apuntan a mejorar las capacidades de liderazgo disponible en el sistema, ya sea asegurando el ingreso al cargo directivo de los candidatos más idóneos (por ejemplo, haciendo más atractiva la carrera directiva o fortaleciendo los procesos de selección), o bien desarrollando habilidades (y prácticas) de liderazgo efectivo en los directivos en ejercicio, principalmente a través de programas de formación (MINEDUC, 2015, p. 4).
\end{abstract}

El MBDLE es el instrumento que define las prácticas, competencias y conocimientos que deben poseer y ejercer quienes asumen cargos directicos en las escuelas municipales del país y, por tanto, las bases de los concursos públicos están regidos por este Marco. Se subdivide en 5 dimensiones:

1) Construyendo e implementando una visión estratégica compartida.

2) Desarrollando las capacidades profesionales.

3) Liderando los procesos de enseñanza y aprendizaje.

4) Gestionando la convivencia y la participación de la comunidad escolar.

5) Desarrollando y gestionando el establecimiento escolar

Fígura 1Marco para la Buena Dirección y el Liderazgo Escolar

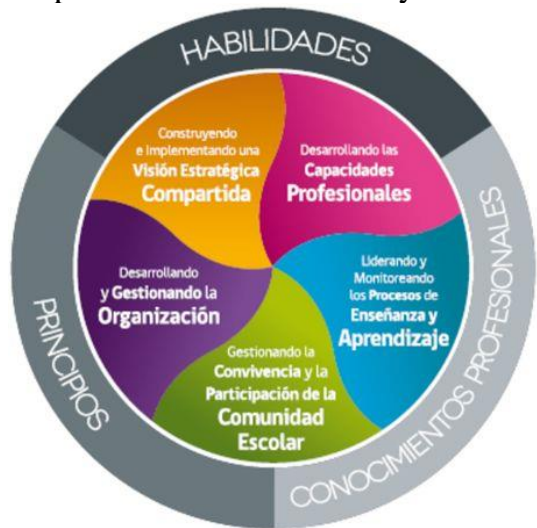

Fuente MINEDUC (2015). Marco para la Buena Dirección y el Liderazgo Escolar. 
Por competencia, el MBDLE define una "una capacidad movilizada, en que se distinguen habilidades, conocimientos y actitudes relacionadas a un desempeño efectivo” (p.9). De allí que el esfuerzo consista en determinar y fijar dichos desempeños efectivos para el mejoramiento escolar. Si bien el MBDLE no es claro en señalar en función de qué y para qué los desempeños serían efectivos, se puede inferir desde la lógica del sistema educativo chileno actual, que ello se mide con indicadores objetivos, por ejemplo, a través de la mejora de los rendimientos en pruebas estandarizadas nacionales (SIMCE, PTU), o los resultados de los docentes en el sistema de evaluación que acredita el desarrollo de sus capacidades profesionales, o la existencia de un Proyecto Educativo Institucional (PEI) y proyectos que lo implementen que evidencia la visión estratégica compartida, o que la gestión del establecimiento sea adecuada para evitar problemáticas de convivencia, recursos u otras.

Si bien, el MBDLE señala que la propuesta excede solo la definición de competencias hacia una postura más contextualizada, a través de la noción de dimensiones de la práctica directiva, en la operacionalización de la política cada dimensión es llevada a competencias y a un descriptor, las que son utilizadas en los concursos públicos como criterio al que se le asigna un porcentaje, como queda en evidencia en la Tabla $\mathrm{N}^{\circ}$ 2. Ello sucede porque, si bien en el MBDLE se declaran las limitaciones del enfoque de competencias debido al foco individual, sin consideración de las interacciones, la fragmentación de los procesos, la descontextualización de experiencias profesionales previas y que no necesariamente consideran los aspectos valóricos, afectivos y éticos necesarios (MINEDUC, 2018, p.10), de todas maneras se adscribe a dicha perspectiva, pues supuestamente el enfoque de competencias ha "logrado sistematizar las capacidades necesarias de los directivos para desarrollar procesos de mejoramiento en sus establecimientos" (MINEDUC, 2018, p. 10).

Por su parte, se intenta complementar el enfoque de competencias incorporando como contextualización ciertos descriptores de "prácticas de liderazgo efectivas y del desarrollo de estándares de desempeño o marco de prácticas que orienten su trabajo, desarrollo profesional y evaluación" (MINEDUC, 2018, p.8). No obstante, finalmente predominan en la formulación final las mismas limitaciones que se pretenden superar, entre otras cosas porque es usado para selección del personal directivo y para la rendición de cuenta, lo que obliga a adoptarlo para evaluar individual, fragmentada y cuantitativamente el desempeño directivo, como queda en evidencia en el ejemplo de propuesta de perfil de cargo para directores, que puede descargarse de Portal Directores para Chile del Ministerio de Educación (Ver tabla N²). 
Tabla $\mathrm{N}^{\circ} 2$ Competencias y su descripción que se consideran en los concursos públicos

Competencias Visión estratégica
compartida

\section{Descripción}

Porcent aje

Construye una visión futura creativa y compartida, que incorpore en su definición a la comunidad, que fomente el compromiso con los objetivos grupales, las altas expectativas, la excelencia y la calidad en el cumplimiento de metas, en un marco de inclusión y equidad. Esta visión deberá ser enfocada en el mejoramiento de los logros de aprendizaje y de la formación integral los niños y niñas como fin último. Incluye la capacidad de conocer y analizar el contexto del establecimiento, de la sociedad actual y como en la construcción de esta visión es integrado el contexto en pro de los logros y resultados.

Desarrollar las
capacidades
profesionales

Capacidad para identificar, comprender, potenciar y articular las habilidades y motivación de docentes y asistentes de la educación, de manera de construir/desarrollar capacidades internas y de equipo que permitan alcanzar objetivos y metas del establecimiento sosteniéndolas en el tiempo. Además, es capaz de detectar oportunamente las necesidades de fortalecimiento para buscar oportunidades de desarrollo profesional y promueve el liderazgo en otros miembros del equipo directivo y docente.

Liderar los procesos Capacidad para gestionar las prácticas asociadas a la sala de clases y de enseñanza y monitoreo de lo que ocurre en ella, demostrando habilidad de proveer de aprendizaje apoyo técnico a docentes. Implica el acompañar la implementación curricular, las prácticas pedagógicas y la evaluación de los logros del aprendizaje, de manera de mantener el foco en los procesos de enseñanza aprendizaje, además de promover el trabajo colaborativo de $\mathrm{y}$ entre los docentes y el equipo directivo. Manifiesta interés en mantenerse actualizado en sus conocimientos y habilidades, en pro de la mejora de los procesos de enseñanza y aprendizaje.

Gestionar la
convivencia:
participación de la
comunidad escolar y
gestión de redes

Capacidad para generar relaciones internas y externas a la escuela que se caractericen por ser colaborativas y que promuevan activamente una convivencia escolar, el alcanzar las metas del Proyecto Educativo Institucional (PEI) y un clima escolar basado en relaciones de confianza y respeto. Incluye la habilidad de comunicarse de manera efectiva, abierta, clara y congruente, en el sentido de poder identificar y establecer estas redes (internas y/o externas) que permitan desarrollo.

Desarrollar y
gestionar el
establecimiento
escolar

Establecer condiciones de trabajo que permitan a los docentes y asistentes de la educación desarrollar todas sus motivaciones y capacidades, gestionando el establecimiento en el marco normativo y político actual, vinculándolo con el sistema en su conjunto y gestionando los recursos de forma eficaz. Con el fin de potenciar las condiciones de calidad de vida dentro del establecimiento, el director debe hacer frente a situaciones complejas o posibles conflictos con estrategia, método y decisión.

Fuente: Propuesta de perfil basado en el Marco para la Buena Dirección y el Liderazgo Escolar. Portal directores para Chile. Disponible en: https://www.directoresparachile.cl/pub/contenido/fuentesLegales.aspx

Dichas competencias y descriptores de prácticas efectivas se fundamentan en una serie de supuestos que se ponen en jaque en las escuelas que se encuentran al interior de los centros penitenciarios o centros de menores, pues en ese contexto las escuelas no gozan de plena autonomía para funcionar, pues deben regirse por lineamientos de Gendarmería y SENAME, incluso con metas a cumplir en materia educativa.

Además, el funcionamiento cotidiano no depende solo de los equipos directivos, por ejemplo, en relación con la asistencia de los internos/as a clases, la superposición de actividades en horarios escolares 
(talleres, trabajo, u otros), suspensión de actividades por allanamientos e incluso paros funcionarios, etc. También hay aspectos simbólicos y de la misión de las instituciones que hacen que la labor de los equipos directivos se vea sometida a otras condicionantes específicas, por ejemplo, la visión de la educación como beneficio, la anteposición de la misión de la seguridad por sobre la educación, miradas y perspectivas de la educación para la rehabilitación o reinserción, la finalidad de la tarea educativa para evitar la reincidencia. Todo lo anterior incide en una serie de medidas, incluso, de quienes se autoriza para asistir a la escuela.

En materia de infraestructura los colegios dependen de otra institución para funcionar, pues no siempre tienen un sector habilitado como escuela con salas y oficinas, sino que los y las docentes deben hacer clases en lugares improvisados o al interior de los pabellones, mientras ocurre la vida cotidiana de los demás internos/as que no están en la escuela, o simplemente porque no cuentan con equipos computacionales, internet, teléfono, biblioteca. etc. o por la alta rotación de estudiantes en las aulas, los que nunca se está seguro si completarán su proceso escolar.

Obviamente, liderar una escuela bajo estas condiciones implica desafíos, competencias, prácticas diferentes de quienes lo hacen en escuelas que cuentan con las condiciones supuestas como mínimas en el MBDLE. En este sentido, se puede advertir algunas tensiones para que los equipos directivos puedan cumplir con las competencias, conocimientos y prácticas que se les exige. Nos centraremos solo en dos que nos parecen claves.

\subsection{MOVILIZAR METAS COMPARTIDAS Y GARANTIZAR LA LABOR PEDAGÓGICA}

La comprensión del liderazgo como "la labor de movilizar e influenciar a otros para articular y lograr los objetivos y metas compartidas" (p.7), así como la atribución a "los equipos directivos (...) de la generación de condiciones organizacionales para garantizar que las actividades pedagógicas se puedan desarrollar" entran en tensión, entre otras cosas, porque los objetivos y metas compartidas en lo estructural, van más allá del cuerpo docente y estudiantil, ya que trasciende a otra institución cuyo objetivo no es educativo y de la cual depende casi el 100\% la posibilidad de funcionamiento de la escuela. En ese mismo sentido, los equipos directivos tienen dificultades para garantizar, incluso, que las clases se lleven a efecto. Subyace a estas tensiones la pregunta si la escuela en contexto de encierro debe "encerrarse" en si misma o bien ampliar la noción de influencia al espacio penitenciario. Desde los datos, podemos señalar que los directivos en contextos de encierro no solo tienen su mirada al interior de la escuela, sino que están compelidos por el contexto a ampliar su ámbito de influencia a otra institución, la penitenciaria, que implica otros ángulos de mirada, prácticas e incluso luchas, por la educación como derecho humano inalienable, que implica otra impronta en quienes realizan la tarea directiva. 


\subsection{INCIDIR EN LAS MOTIVACIONES Y PRÁCTICAS DE LOS/AS DOCENTES}

La asunción de MBDLE de "la incidencia de los directivos en ámbitos como la motivación, las habilidades, prácticas y condiciones de trabajo en que se desempeñan los docentes" (p.8) también constituye un ámbito que requiere de otras consideraciones diferentes a las escuelas del medio libre, entre otras cosas, porque se exige que los equipos directivos desarrollen "estrategias de retención para mantener un cuerpo docente estable, motivado y comprometido, a través del mejoramiento de sus condiciones laborales y la generación de un clima de confianza y desafiante profesionalmente" (p.22), no obstante, la cárcel es un escenario desgarrador que no se puede soslayar de la experiencia docente, es decir, no solo se hace clases --¡Se hace clases en la cárcel!- Cuestión que es vital para las motivaciones y prácticas de los docentes, sobre todo en un país donde hay escasa formación especializada y donde el sistema de evaluación docente basado en estándares no considera que hay ambientes socioprofesionales que no dependen de las escuelas pero que condicionan sus prácticas. Al respecto, hay cuestiones que dependen de otra institución que condicionan los desempeños y motivaciones de los y las docentes, por ejemplo, en relación con el trato que reciben, la comprensión del trabajo que realizan, las posibilidades de colaboración, el tipo de peligrosidad de las y los internos.

Además, se debe considerar que las prácticas de las y los docentes se ven afectadas por los procesos de prisionización (Arroyo, López y Lacal, 2004) y, que no solo afectan a las y los internos/as, sino a los profesionales que viven el encierro, en ocasiones 8 horas diarias por 5 días a la semana, lo que va dejando huellas en la subjetividad (por ejemplo, desconfianza, miedo, asunción de la cultura carcelaria, etc.), afecta las motivaciones y condiciona las prácticas. Además, se debe tener presente que al interior de la cárcel trabajan distintos profesionales que deben coordinarse dentro del espacio para ejercer su labor, cuestión que es compleja y puede causar tensiones y conflictos entre personal de distintas instituciones que se orientan por objetivos distintos, lo que también puede incidir en las motivaciones y prácticas de las y los docentes.

El carácter multiprofesional (...) puede conllevar conflictos -prejuicios grupales, estereotipos, modelos teóricos distintos, formación diferente, distintas categorías profesionales y posibilidades de promocionar, dificultades de planificación y relación, etc.- entre grupos corporativos, junto al temor de todos a perder autonomía, recursos y autoridad. (...) Todo esto hace que el personal del medio penitenciario tenga que afrontar un difícil reto en la coordinación efectiva de sus actuaciones y, si ésta no se realiza adecuadamente, sufra el riesgo del desencanto o la fatiga en la tarea a desarrolla (del Mar García-Vita, y Melendro, 2013, p. 47) 


\section{LOS SUEÑOS DOTAN DE CONTENIDO LAS PRÁCTICAS DE LIDERAZGO EDUCATIVO EN CENTROS DE MENORES Y PENITENCIARIOS}

Los dos puntos desarrollados no tienen el propósito de afirmar que nada se puede hacer en las escuelas en contextos de encierro, todo lo contrario, los equipos directivos y las comunidades hacen mucho, pero orientados por otras necesidades y objetivos, que exceden el MBDLE, donde consideran la crudeza del contexto, la precariedad de los recursos e infraestructura, la dependencia de su funcionamiento cotidiano a otra institución, las huellas que va dejando en ellos el dolor que presencian, entre otros.

Leer el MBDLE nos lleva a imaginar un cuerpo directivo más bien gerencial, ello porque "gerenciar es administrar procesos para lograr metas y objetivos (...) Es lograr los objetivos de la empresa para que este alcance su máximo rendimiento. Es hacer más eficiente y efectiva las acciones en la organización” (Chacón, Briceño, Zavance, 2009, p.90). Desde esta perspectiva, la orientación gerencial del liderazgo tiene su foco en la tarea, en la productividad, el rendimiento, la eficiencia, la eficacia y la efectividad. Por tanto, quien guía su accionar por esos propósitos puede dirigir diversas instituciones a lo largo de su vida, incluso por cortos periodos de tiempo, y en cada uno tendría que desempeñarse en forma destacada, en la medida que las habilidades directivas que poseen podrían ponerse en ejecución en diversos contextos, aunque estos tengan condicionantes culturales, implicancias éticas y políticas o anclajes sociohistóricas diferentes. Así el contenido profundo que moviliza las acciones de liderazgo, y que responde a las cuestiones de para qué liderar, a favor de quién, tras qué intereses de poder, etc. no es considerado.

No obstante, el liderazgo gerencial contrasta con la realidad de las escuelas en las cárceles. Paradójicamente podría ocurrir que una persona que ha desarrollado todas las capacidades y competencias señalas por MBDLE podría no desempeñarse del todo bien en un contexto tan adverso, donde antes que planificar estratégicamente, cautelar el proceso de enseñanza y aprendizaje, potenciar el desarrollo profesional docente, gestionar la convivencia y la participación, debe luchar por la educación como derecho, hacer escuela en cualquier espacio, preocuparse por la exclusión estructural que han vivido sus estudiantes, gestionar la precariedad (a pesar de todo), aceptar la incertidumbre, flexibilizar al máximo los procesos y planes de acción, conquistar a la institución carcelaria para que mueva los limites del paradigma de la seguridad al de la educación, jaquear los procesos de prisionización, ampliar la noción de enseñanza y aprendizaje más allá de los rendimientos aceptables, desnaturalizar lo que se considera en una escuela como prácticas efectivas, resignificar el espacio pedagógico desde principios de la educación popular (que parecen con mayor pertinencia); en fin, poner en cuestión el sentido de la educación escolar excluyente y reproductora de las desigualdades sociales, de las que son testigos cada día. 
Desde la óptica anterior se pueden encontrar muchas más semejanzas entre los equipos directivos de las escuelas con aquéllos que están a cargo de proyectos de educación no formal, como fundaciones, ONG, corporaciones e incluso con quienes lideran los colectivos o voluntariados universitarios que con directores de escuelas que corporizan el perfil del líder gerencial que se infiere del MBDLE. Entre aquellas cuestiones comunes encontramos el liderazgo como:

1. Lucha por el Derecho a la Educación.

2. Resignificación de la educación y la escuela desde el acto de formación entre un profesor y un estudiante se dé donde se dé

3. Conciencia de la relevancia del acto de educar para esos sujetos privados de libertad

4. Subordinación del currículo, los planes, las metas u otros a los sujetos/as y no viceversa

5. Conciencia del rol social, moral o político del trabajo que se realiza y su posible impacto en la sociedad en que vivimos

6. Convicción que la escuela o el espacio de formación, en el caso de los proyectos de educación no formal, no solo no es igual que la cárcel, sino que se constituye en un espacio de libertad.

7. Una tarea que se realiza al servicio de los otros/as, donde muchas veces, hay que poner el propio bienestar entre paréntesis, flexibilizar las reglas, saltar las normas que no son pertinentes, decidir en delgados límites, incluso contravenir los llamados institucionales o de quienes financian por la efectividad, el rendimiento y la eficacia en favor de las necesidades integrales de los internos e internas

8. Problematizar, cuestionar, o resistir las orientaciones de la educación chilena que es altamente desigualdad intentando construir junto a los equipos nuevos sentidos.

Las dimensiones anteriores evidencian un contenido especifico y determinante de las acciones que emprenden cada día los equipos directivos escolares y de educación no formal. Dichas dimensiones constituyen parte del "sueño pedagógico" de los equipos que conducen las escuelas o programas de educación no formal, tal y como plantean algunos/as entrevistados/as:

hay que soñar y hay [que] lanzarse también. (Directora, proyecto formación en oficios en centro de protección de menores).

Nuestro sueño es que este estudiante que salga del CIP CRC cuando vaya a un colegio, entre comillas, tradicional tenga las mismas herramientas realmente, si no pensamos así vamos a estar siempre pensando que este estudiante es un estudiante distinto (Director de Escuela).

Los directivos se caracterizan por un fuerte compromiso ético o moral, que también se lo exigen a quienes postulan a trabajar en las escuelas o proyectos que dirigen, tal y como plantean diversos directores de proyectos no formales y directores de escuelas:

Definimos bien un perfil para quienes quieran trabajar con nosotros, qué es $100 \%$ comprometido con la causa, en el fondo, establecer que aquí nadie me puede abandonar a los jóvenes, o sea, un abandono más jyo no se los voy a dar! (Directora, proyecto formación en oficios en centro de protección de menores).

Hoy en la mañana hablaba con un profesor que fue instalado por el DAEM, y me dijo: —director yo no tengo el perfil porque hay que tener un perfil distintivo-. Primero una alta vocación, un 
alto compromiso, porque el profesor que no cree en los chicos se ve, ellos lo huelen... (Director de colegio en cárcel de menores).

el trabajo en contextos de encierro (...) es un tema que tiene que ver con el compromiso de un profesor de atender a la diversidad de manera justa, equitativa ir brindando procesos de calidad (Académica Universidad, directora proyecto educación en cárceles).

Consecuentemente, los equipos directivos no dudan en ir más allá de lo que les corresponde y buscar alternativas autogestionadas para realizar su labor. Lo anterior posibilita que los proyectos no formales y las escuelas se constituyan en un espacio simbólico muy diferente al espacio carcelario, alejándose de la lógica de vigilar y castigar.

\section{CONCLUSIONES}

Liderar una organización educativa implica desafíos que exceden con creces el liderazgo gerencial, pues la finalidad no es que la institución alcance mayor efectividad, eficacia, o rendimiento, sino de qué manera se emprenden acciones con sentido formativo para los y las sujetas que no solo están aprendiendo ciertos contenidos sino configurando su subjetividad en esos espacios de formación.

Esta reflexión no es del todo evidente en las escuelas de nuestro país, que más bien están orientadas a fortalecer la propia institución, más que avocadas al desarrollo humano, la justicia social, el derecho inalienable a la educación, lo que queda en evidencia por los sistemas de expulsión actuales, los que se pretenden fortalecer aún más. No obstante, en los contextos de encierro esta cuestión se vuelve evidente, de modo, que los lideres escolares más que guiar sus acciones por competencias que podrían estar al servicio de ciertos intereses de poder que reproducen la desigualdad social, centran sus esfuerzos directivos en una "causa”, con la que podríamos o no estar de acuerdo, que dota de contenido su labor, y la que sirve de guía en sus prácticas directivas y toma de decisiones. De este modo, desde los directivos de las escuelas hasta los voluntarios de colectivos tiene mucho en común: Un contenido que dota de sentido sus acciones. 


\section{REFERENCIAS}

Arroyo, J. M., López, J. L. y Lacal, P. (2004). Problemas psicológicos y trabajo en prisión. Revista Española Sanidad Penitenciaria, 6, 47-56.

Chacón, M., Briceño, M, y Zavance, C. (2009). La gerencia en las organizaciones sociales: perspectiva crítica interpretativa. Revista Negotium, 12, 86-99.

del Mar García-Vita, M, y Melendro, M. (2013). El ambiente en prisión: La atención recibida por las reclusas y las relaciones intramuros. Pedagogía social: revista interuniversitaria, 22, 4-14.

MINEDUC (2015). Política de fortalecimiento del liderazgo directivo escolar 2014-2017. Santiago: Unidad de Liderazgo Escolar. División de Educación General. Disponible en: https://liderazgoescolar.mineduc.cl/wp-content/uploads/sites/55/2018/01/Politica-Fortalecimiento-delLiderazgo-Escolar-2014-2018_Enero_208.pdf 